\title{
AB INITIO CALCULATIONS OF RESPONSE PROPERTIES INCLUDING THE ELECTRON-HOLE INTERACTION
}

\author{
VALERIO OLEVANO*,**,STEFAN ALBRECHT*, LUCIA REINING* \\ * Laboratoire des Solides Irradiés, UMR 7642 CNRS - CEA, École Polytechnique, F-91128 \\ Palaiseau, France \\ ** Istituto Nazionale per la Fisica della Materia, Dipartimento di Fisica dell'Università \\ di Roma "Tor Vergata", Via della Ricerca Scientifica, I-00133 Roma, Italy
}

\begin{abstract}
We discuss the current status of a computational approach which allows to evaluate the dielectric matrix, and hence electronic excitations like optical properties, including local field and excitonic effects. We introduce a recent numerical development which greatly reduces the use of memory in such type of calculations, and hence eliminates one of the bottlenecks for the application to complex systems. We present recent applications of the method, focusing our interest on insulating oxides.
\end{abstract}

\section{INTRODUCTION}

Density-functional theory (DFT) in the Local Density Approximation (LDA) is widely and successfully used as a state-of-the-art tool to compute the ground-state electronic properties of many-electron systems [1]. It is feasible to apply DFT to systems as complex as surfaces, defects, and clusters. However, while the ground-state properties can in principle be obtained exactly within DFT, many spectroscopic properties are in general not directly accessible in such a calculation.

In fact, it is well known that the use of the DFT-LDA eigenvalues as the physical energies entering in the absorption process relies on several approximations. First of all, Kohn-Sham LDA eigenvalues are only a rough approximation of the electron addition or removal energies, such those measured in photoemission. The correct energies should be obtained by using the true electron self-energy operator $\Sigma$, which appears at the place of the DFT-LDA exchangecorrelation potential in an equation similar to the Kohn-Sham one. A good approximation for $\Sigma$, which allows to determine the quasiparticle (QP) bandstructure, can be obtained within Hedin's GW approach [2]. In this scheme, DFT-LDA results can be used as the starting point, in a first-order perturbative approach [3, 1 .

However, the true QP energies, together with the LDA wavefunctions, are in principle still not sufficient to describe correctly an absorption process, in which electron-hole pairs are created. Their interaction can lead to bound exciton states which occur within the gap, and can also induce appreciable distortions of the spectral lineshape above the continuousabsorption edge.

Since recently [6, 7, 8, 9, 10, 11], these excitonic effects can be treated in the ab initio framework. Various applications have demonstrated the success of the Green's functions approach [6, 7, 8, 9, 10, 11, 12, 13. However, the calculations are still very cumbersome, since a two-particle problem has to be solved. This prevents the method from being applied on a large scale.

In this work, we introduce a perturbative approach to the solution of the Bethe-Salpeter equation which greatly reduces the use of memory in such type of calculations, and hence eliminates one of the bottlenecks for the application to complex systems. We show that the approximation is perfectly controllable, hence the approach is still $a b$ initio. In order to test the limits of the method, we present recent applications for the case of insulating oxides. 


\section{THE APPROACH}

The absorption spectrum is given by the imaginary part of the macroscopic dielectric function $\epsilon_{M}$

$$
\epsilon_{M}(\omega)=1-\lim _{\mathbf{q} \rightarrow 0} v(\mathbf{q}) \hat{\chi}_{\mathbf{G}=0, \mathbf{G}^{\prime}=0}(\mathbf{q} ; \omega),
$$

where $\hat{\chi}\left(\mathbf{r}, \mathbf{r}^{\prime} ; \omega\right)=-i S\left(\mathbf{r}, \mathbf{r}, \mathbf{r}^{\prime}, \mathbf{r}^{\prime} ; \omega\right)$. The four-point function $S$ obeys the Bethe-Salpeter equation,

$$
S\left(1,1^{\prime} ; 2,2^{\prime}\right)=S_{0}\left(1,1^{\prime} ; 2,2^{\prime}\right)+S_{0}\left(1,1^{\prime} ; 3,3^{\prime}\right) \Xi\left(3,3^{\prime} ; 4,4^{\prime}\right) S\left(4,4^{\prime} ; 2,2^{\prime}\right) .
$$

The notation $(1,2)$ stands for two pairs of space and time coordinates, $\left(\mathbf{r}_{1}, t_{1} ; \mathbf{r}_{2}, t_{2}\right)$. Repeated arguments are integrated over. The term $S_{0}\left(1,1^{\prime} ; 2,2^{\prime}\right)=G\left(1^{\prime}, 2^{\prime}\right) G(2,1)$ yields the polarization function of independent quasiparticles $\chi_{0}$, from which the standard RPA $\epsilon_{M}$ is obtained $\left(G\left(1,1^{\prime}\right)\right.$ is the one-particle Green's function [5]). The kernel $\Xi$ contains two contributions:

$$
\Xi\left(1,1^{\prime}, 2,2^{\prime}\right)=-i \delta\left(1,1^{\prime}\right) \delta\left(2,2^{\prime}\right) v(1,2)+i \delta(1,2) \delta\left(1^{\prime}, 2^{\prime}\right) W\left(1,1^{\prime}\right) .
$$

Considering the first term in the calculation of $S$ is equivalent to the inclusion of local field effects in the matrix inversion of a standard RPA calculation. In order to obtain the macroscopic dielectric constant, the bare Coulomb interaction $v$ contained in this term must, however, be used without the long range term of vanishing wave vector [14. When spin is not explicitly treated, $v$ gets a factor of two for singlet excitons. In the second term, $W$ is the screened Coulomb attraction between electron and hole. It is obtained as a functional derivative of the self-energy in the $G W$ approximation, neglecting a term $G \frac{\delta W}{\delta G}$. This latter term contains information about the change in screening due to the excitation, and is expected to be small [15]. We limit ourselves to static screening, since dynamical effects in the electron-hole screening and in the one particle Green's function tend to cancel each other [16], which suggests to neglect both of them. The set of equations (1) - (3) are at the basis of all the $a b$ initio exciton calculations which have appeared in the literature recently [6, 7, 8, 9, 10, 11, 12, 13].

In order to solve Eq. (2), we rewrite it as an effective eigenvalue problem,

$$
\sum_{\left(n_{3}, n_{4}\right)} H_{e x c}^{\left(n_{1}, n_{2}\right),\left(n_{3}, n_{4}\right)} A_{\lambda}^{\left(n_{3}, n_{4}\right)}=E_{\lambda} A_{\lambda}^{\left(n_{1}, n_{2}\right)}
$$

with

$$
\begin{aligned}
H_{e x c}^{\left(n_{1}, n_{2}\right),\left(n_{3}, n_{4}\right)=} & \left(E_{n_{2}}-E_{n_{1}}\right) \delta_{n_{1}, n_{3}} \delta_{n_{2}, n_{4}}-i\left(f_{n_{2}}-f_{n_{1}}\right) \times \\
& \int \cdot \int d \mathbf{r}_{1} d \mathbf{r}_{1}^{\prime} d \mathbf{r}_{2} d \mathbf{r}_{2}^{\prime} \psi_{n_{1}}\left(\mathbf{r}_{1}\right) \psi_{n_{2}}^{*}\left(\mathbf{r}_{1}^{\prime}\right) \Xi\left(\mathbf{r}_{1}, \mathbf{r}_{1}^{\prime}, \mathbf{r}_{2}, \mathbf{r}_{2}^{\prime}\right) \psi_{n_{3}}^{*}\left(\mathbf{r}_{2}\right) \psi_{n_{4}}\left(\mathbf{r}_{2}^{\prime}\right) .
\end{aligned}
$$

The $\psi_{n}(\mathbf{r})$ are LDA Bloch functions, with $n$ denoting a band index and a Bloch vector k. For the calculation of absorption spectra, we can limit ourselves to transitions with positive frequency, i.e. $\left(n_{1}, n_{2}\right)$ and $\left(n_{3}, n_{4}\right)$ are pairs of one valence and one conduction band, respectively (in other words, we consider only the resonant part). Moreover, we build up the spectra of optical properties by considering only negligible momentum transfer, hence the same $\mathbf{k}$ for the valence and the conduction state. Equation (1) reads then:

$$
\epsilon_{M}(\omega)=1+\lim _{\mathbf{q} \rightarrow 0} v(\mathbf{q}) \sum_{\lambda} \frac{\left|\sum_{v, c ; \mathbf{k}}\left\langle v, \mathbf{k}-\mathbf{q}\left|e^{-i \mathbf{q r}}\right| c, \mathbf{k}\right\rangle A_{\lambda}^{(v, c ; \mathbf{k})}\right|^{2}}{\left(E_{\lambda}-\omega\right)} .
$$




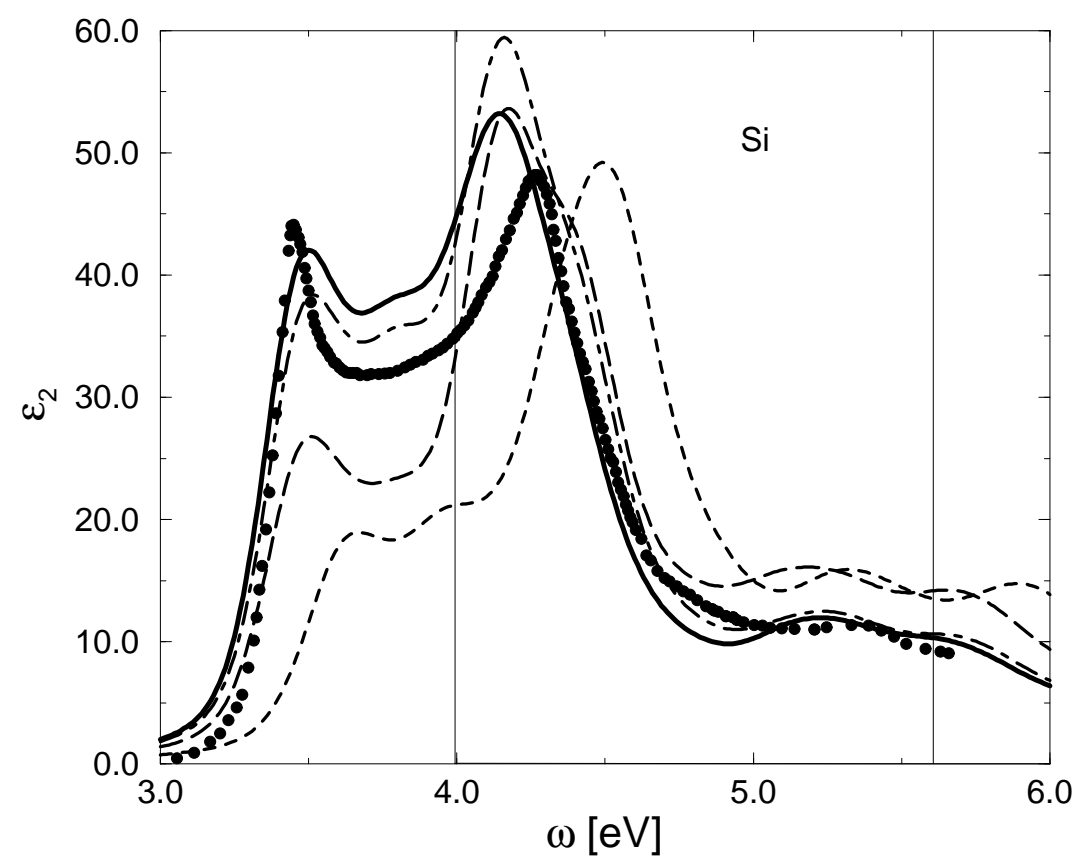

Figure 1: Absorption spectrum of bulk silicon. Dots: Experiment [19]. Short-dashed curve: calculation, no electron-hole interaction included. Continuous curve: full exciton calculation. Long-dashed curve: zero-order result. Dot-dashed curve: first order result. Vertical bars: block cuts.

\section{The problem}

The advantage of this approach with respect to a straightforward inversion of equation (2) is that we have to diagonalize the Hamiltonian (5) only once, and can then construct the spectrum for all frequencies. Moreover, the knowledge of the coefficients $A_{\lambda}^{(v, c ; \mathbf{k})}$ allows for a detailed analysis of the results. However, the matrix to be diagonalized can be very large, since the basis set is built up of pairs of states. Moreover, the number of k-points in the Brillouin zone sampling needed for the calculation of optical properties is typically at least one order of magnitude bigger than that employed in a ground state calculation. Even though this number enters the basis linearly, and not quadratically as the number of bands, the basis is very large, and the calculations are prohibitive for an application to an extended range of energies, i.e. including states far from the Fermi level, or to systems with many atoms.

In order to find a way for overcoming this difficulty, it is worthwhile to analyse how excitonic effects alter the absorption spectra.

We first look at bulk Silicon. The importance of excitonic effects in the absorption spectrum of Silicon have already been shown by Hanke and Sham in a semi-empirical calculation [5]. Recently, ab initio calculations [8, 9] have confirmed their findings. For this work, we have repeated the calculations of Ref. [8], but using a k-point set for the Brillouin zone (BZ) sampling which is shifted off the high symmetry directions, whereas in Ref. [8] special points [17] were used. It has in fact been discussed [18] that the shifted grid gives improved results. Still, the number of k-points which must be used in order to get reasonable results is big; here we have chosen 256 points in the BZ, which, together with the 4 valence bands and the 3 lowest conduction bands, leads to a matrix size of $3072 \times 3072$. (It is obvious that increasing the size of the unit cell or the energy range which is considered would let the problem explode rapidly.) 


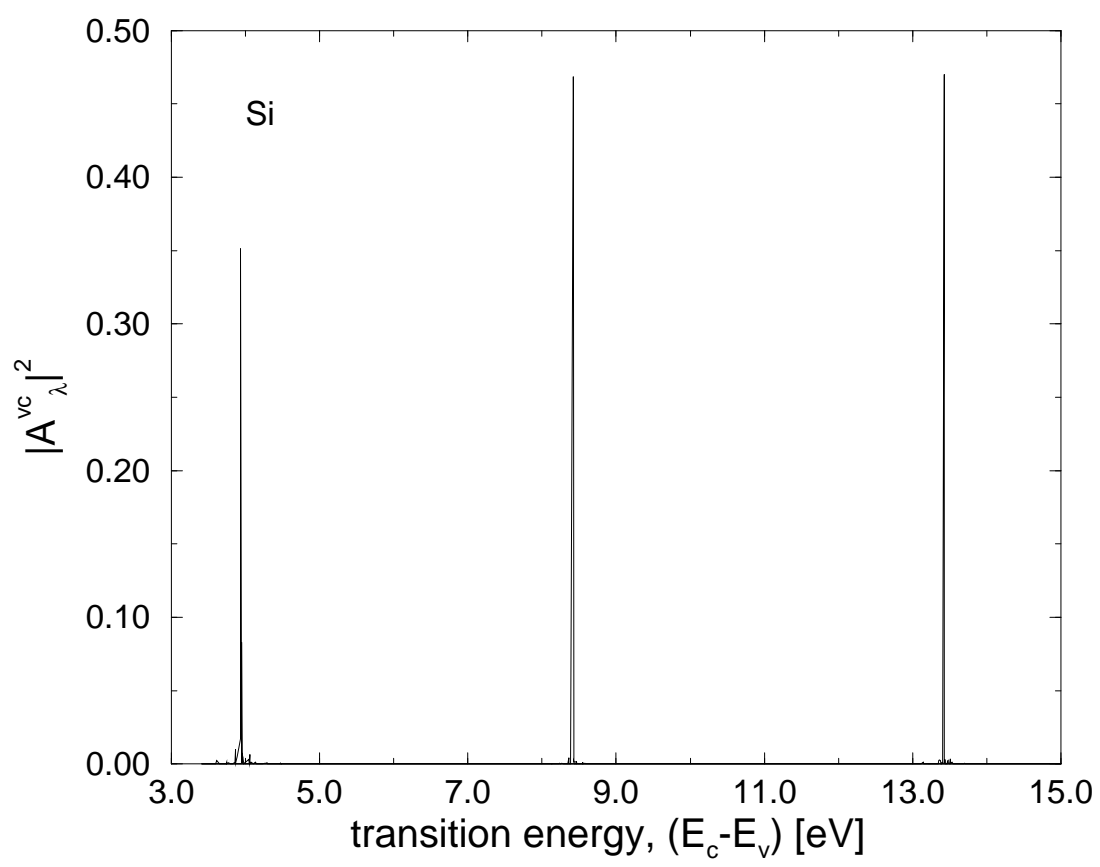

Figure 2: Contribution of independent-quasiparticle transitions to excitonic transitions at given energy (see text), for bulk silicon

\section{Analysis of the excitonic effect}

The continuous curve in Fig. 1 1 shows hence the absorption spectrum of bulk silicon which we obtain using the parameters described above. It is in good agreement with experimental data [19] (dots in Fig. 1), and with the previous ab initio calculations [8, 9]. We can now analyse the origin of the excitonic effects. First of all, the fact that excitonic effects change the peak positions in Silicon can be misleading: in fact, the density of transition energies does virtually not change between the independent quasiparticle and the exciton result. This has been found not only for silicon, but seems to hold quite generally for those materials where no strongly bound excitons exist (see, for example, [12, 13]). The effect is hence entirely due to a change in oscillator strength, in other words, to the coefficients $A_{\lambda}^{(v c)}$ from equation (4). $A_{\lambda}^{(v c)}$ expresses the mixing of different independent quasiparticle transitions with energy $E_{c}-E_{v}$ into a given transition with energy $E_{\lambda}$. Fig. 2 shows the absolute squared value $\left|A_{\lambda}^{(v c)}\right|^{2}$ for three different $\lambda$, with $E_{\lambda}$ chosen above the independent quasiparticle gap by $0.5 \mathrm{eV}, 5 \mathrm{eV}$ and $10 \mathrm{eV}$, respectively, in function of the energy of the contributing independent-quasiparticle transitions, $E_{c}-E_{v}$. One can notice that the distributions are extremely sharp; in fact more than $90 \%$ of the weight is contained in an energy range around each peak position of less than $0.1 \mathrm{eV}$, and more than $99 \%$ of the weight is contained in a region of less than $1 \mathrm{eV}$. This suggests at first sight that one should divide the spectrum into several parts, and for each energy range carry out the calculations using the corresponding limited part of the hamiltonian only.

\section{The perturbative approach}

In other words, one could hope that a separate diagonalisation of several subblocks, neglecting the interaction between the blocks, could yield a satisfactory absorption spectrum for all energies except those very close to the boarder of the blocks. This is however not true: In 
Fig. 1 we compare the result obtained by exact diagonalisation of the full $3072 \times 3072$ Hamiltonian (continuous curve) with that obtained by choosing about 40 blocks in a reasonable way, i.e. by avoiding to cut at energies close to maxima in the density of states. The result is given by the long-dashed curve. The vertical lines indicate the block cuts. The agreement of the result obtained in this way with the full calculation is not satisfactory at any energy; in particular, when we compare with the result obtained by neglecting the electron-hole interaction (short-dashed curve) we notice that a big part of the excitonic effect has been lost. In order to obtain the full effect, it is hence unavoidable to let the states interact even at relatively large energy distances. We can however assume that the interaction decreases with increasing energy separation, and try to include the missing contributions in a perturbative approach, as also suggested in Ref. 20.

In fact, we can treat that portion of the excitonic hamiltonian, which has been neglected up to now by our choice of the blocks, in first order perturbation theory for quasi-continuum states. In this way, we obtain the dot-dashed curve in Fig. 1: the agreement with the full result is now very good. Note that in this way, we have to diagonalize matrices of a maximum size of $100 \times 100$, whereas the full calculation requires the diagonalization of a $3072 \times 3072$ matrix. This represents a memory saving of about three orders of magnitude, which will be very useful for the study of bigger systems, or in order to be able to improve the k-point sampling. It must be stressed that the quality of the resulting spectrum can be checked and systematically improved by increasing the block size of the zero-order calculation.

\section{RESULTS FOR INSULATING OXIDES}

This new approach looks hence extremely promising, and it can be expected that the method will work similarly well for systems with the same behaviour as silicon: silicon has in fact the peculiarity that screening is strong, hence the electron-hole interaction matrix elements $W_{v c}^{v^{\prime} c^{\prime}}$ are weak. The strong deformation of the spectrum comes from the fact that many transitions at energies very close to each other, where

$W_{v c}^{v^{\prime} c^{\prime}} /\left[\left(E_{c}-E_{v}\right)-\left(E_{c^{\prime}}-E_{v^{\prime}}\right)\right]$ is big, are interacting. This point is of course very favourable for our approach, and it is interesting to explore whether the method can also be applied in a less favourable situation. In order to become more severe, we can choose an insulator, with low dielectric constant, and in the following we will hence look at two insulating oxides: $\mathrm{MgO}$ and $\mathrm{Li}_{2} \mathrm{O}$.

\section{$\mathrm{MgO}$}

Excitonic effects in the absorption spectrum of magnesium oxide have been calculated $a b$ initio by Shirley et al [10], using the Haydock recursion method [21] for a direct solution of equation (2). Excitonic effects have turned out to alter the absorption spectrum significantly. With a dielectric constant of only about $\epsilon_{M}=3, \mathrm{MgO}$ is hence a good candidate for our test. We have performed the ground state calculations using pseudopotentials of the Trouiller-Martins type [22], a plane-wave cutoff of $50 \mathrm{Ry}$, and using 10 special points in the irreducible part of the BZ. The theoretical lattice constant is $4.13 \mathrm{~A}$ in good agreement with the experimental value of $4.21 \mathrm{~A}$. We have then determined the GW corrections to the DFT-LDA eigenvalues, using 307 reciprocal lattice vectors and 150 bands. The correction to the direct gap turns out to be $2.5 \mathrm{eV}$, and the corrections are slightly larger at the zone boundaries, in good agreement with [23] and [24]. Our independent quasi-particle absorption spectrum, calculated using 256 shifted k-points in the BZ and the first 9 bands, (dotted curve in Fig. 3) is close to the result of Ref. [10], and in very bad agreement with experiment (dots in Fig. 3) [25]. We have then calculated the full exciton absorption spectrum according to equation (6), using the same parameters as for the independent quasiparticle spectrum. The result is given by the continuous curve in Fig. 3, and is again close to the findings of Ref. [10]. As in that work, the very strong excitonic effect significantly improves the agreement with experiment [25, 26]. The main discrepancy is the overestimation of the peak intensities, which might be due to our limited k-point sampling. Now we want to try 


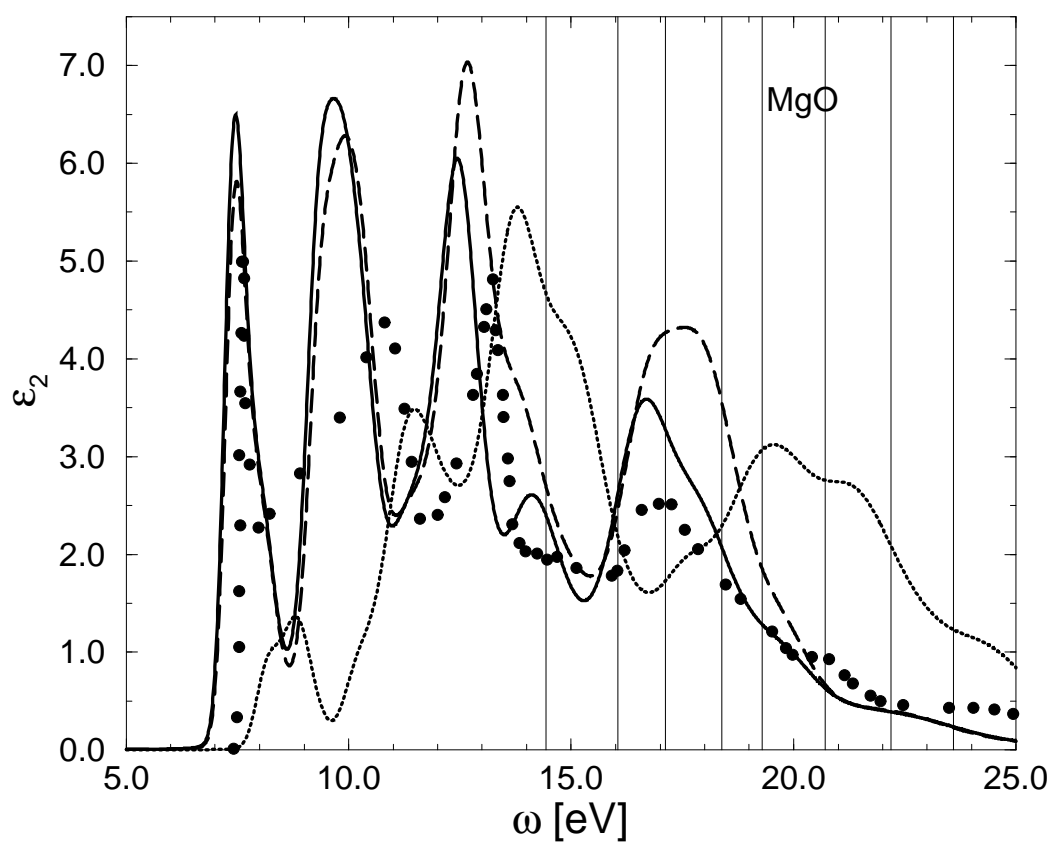

Figure 3: Absorption spectrum of magnesium oxide. Dots: Experiment 25]. Dotted curve: calculation, no electron-hole interaction included. Continuous curve: full exciton calculation. Dashed curve: first order result. Vertical bars: block cuts.

our perturbative approach. First, we can check if this might be reasonable, by analysing the exciton eigenstates as we did in the case of silicon. This analysis is shown in Fig. 4. Also in $\mathrm{MgO}$, the distributions are looking rather sharp, but of course, due to the stronger $W_{v c}^{v^{\prime} c^{\prime}}$, transitions from a wider range of the spectrum must be taken into account. This time, about $90 \%$ of the weight of the peaks is contained in a range of less than $1.5 \mathrm{eV}$, and in order to retain $99 \%$ of the weight we must go up to almost $7 \mathrm{eV}$ for the second peak ( $3 \mathrm{eV}$ for the first one and $2 \mathrm{eV}$ for the third one). This tells us that we certainly have to choose bigger blocks than in Silicon in order to obtain reasonable results. However, still we will try to reduce the memory requirement by about two orders of magnitude, and therefore we choose the block borders as indicated by the vertical lines in Fig. 5. This corresponds to blocks of a maximum size of $500 \times 500$. The resulting first-order spectrum is given by the dashed curve. Again, the agreement with the full calculation is good, and most of the disagreement between the independent quasiparticle and the experimental result has been removed. The most important errors occur of course at higher energies, where the states are denser and hence the energy range of the blocks smaller. Due to the Kramers Kronig relation, one could suspect that this fact might spoil the real part of $\epsilon_{M}$ even at low energies, and hence show up in spectra linked to both the real and the imaginary part, like reflectance. We have therefore used our results in order to calculate the reflectance spectrum of $\mathrm{MgO}$. The independent quasiparticle result is given by the dotted curve in Fig. 5, and the result of the full exciton calculation is given by the continuous curve. As in the case of $\operatorname{Im}\left(\epsilon_{M}\right)$, the inclusion of the electron-hole interaction has a dramatic effect. In particular, the weak structures up to 15 $\mathrm{eV}$ in the GW spectrum are transformed into pronounced peaks, which are consistent with the experimentally found structures in that energy range in the reflectance spectrum (see inset) [25]. The first-order result, for the block size of $500 \times 500$, is given by the dashed curve. Again, the agreement with the full calculation at lower energies is excellent, and also the structures at higher energies are considerably improved with respect to the $\mathrm{GW}$ calculation. The performance of the method for $\mathrm{MgO}$ is hence less spectacular than in the case of silicon, but still the approach turns out to be valid. 


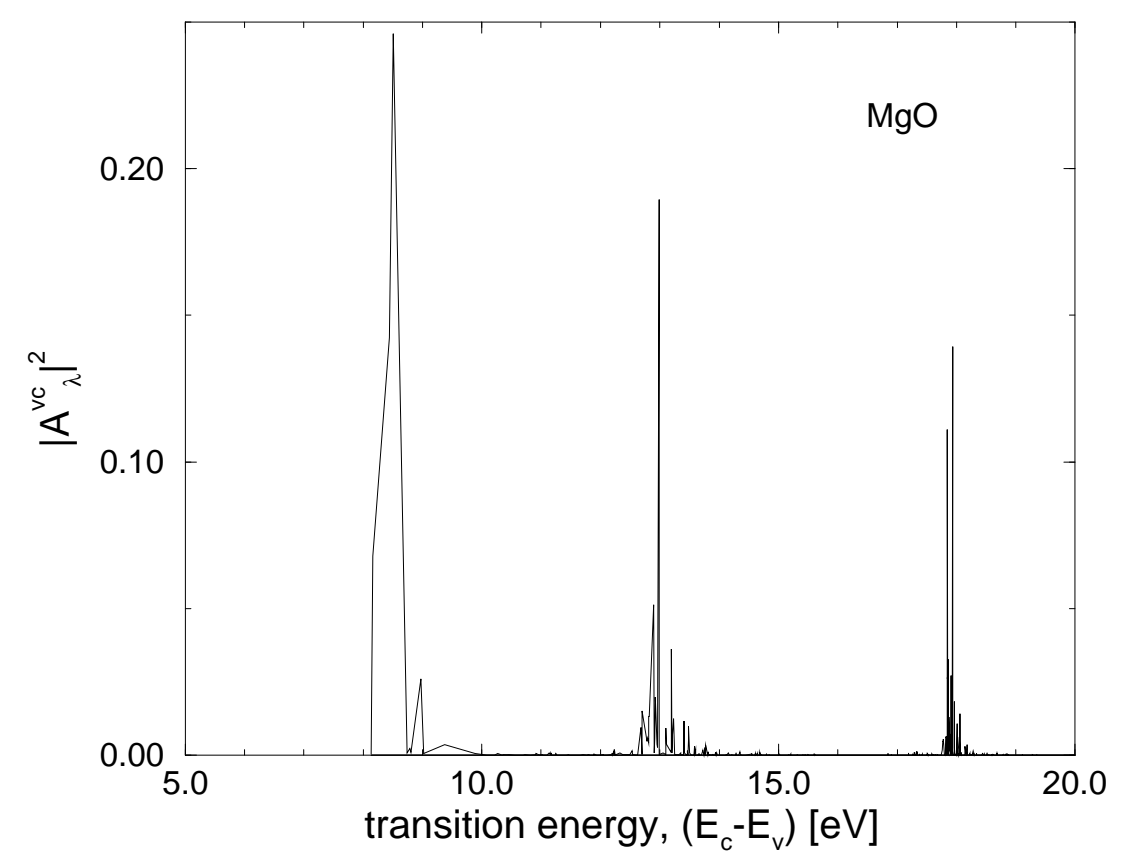

Figure 4: Contribution of independent-quasiparticle transitions to excitonic transitions at given energy (see text), for magnesium oxide

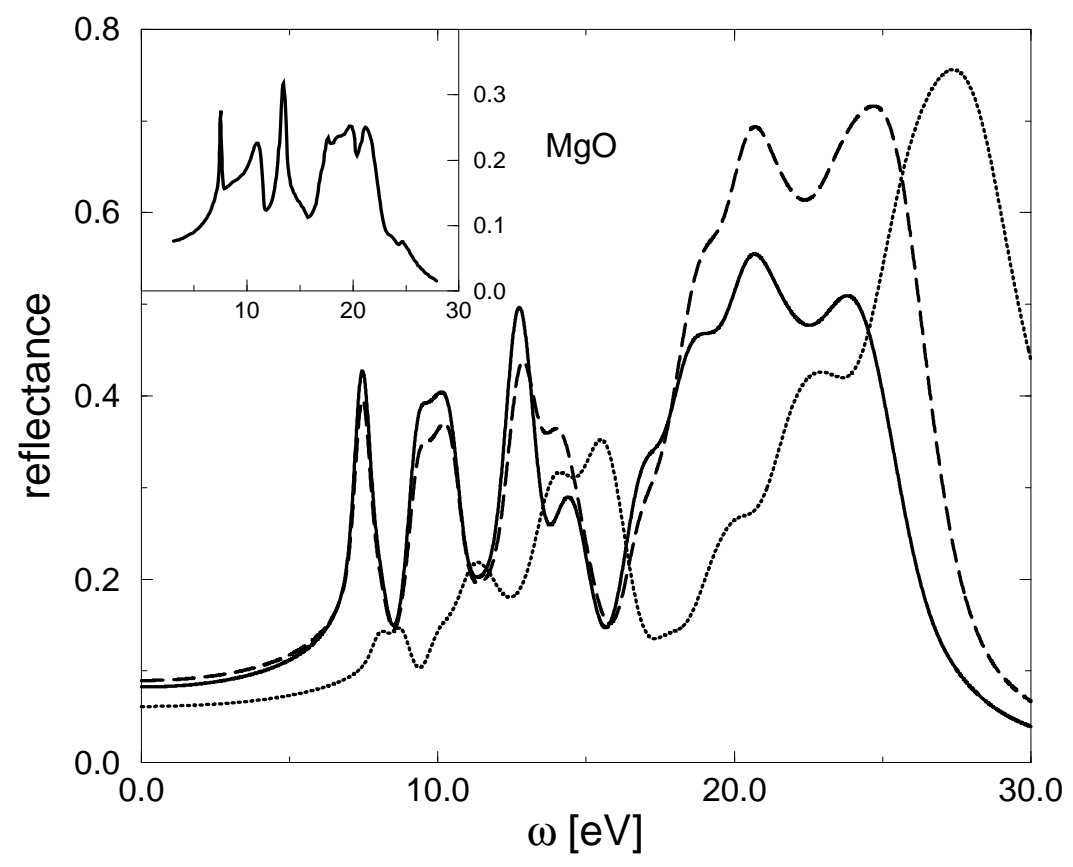

Figure 5: Reflectance spectrum of magnesium oxide. Inset: Experiment 25]. Dotted curve: calculation, no electron-hole interaction included. Continuous curve: full exciton calculation. Dashed curve: first order result. 


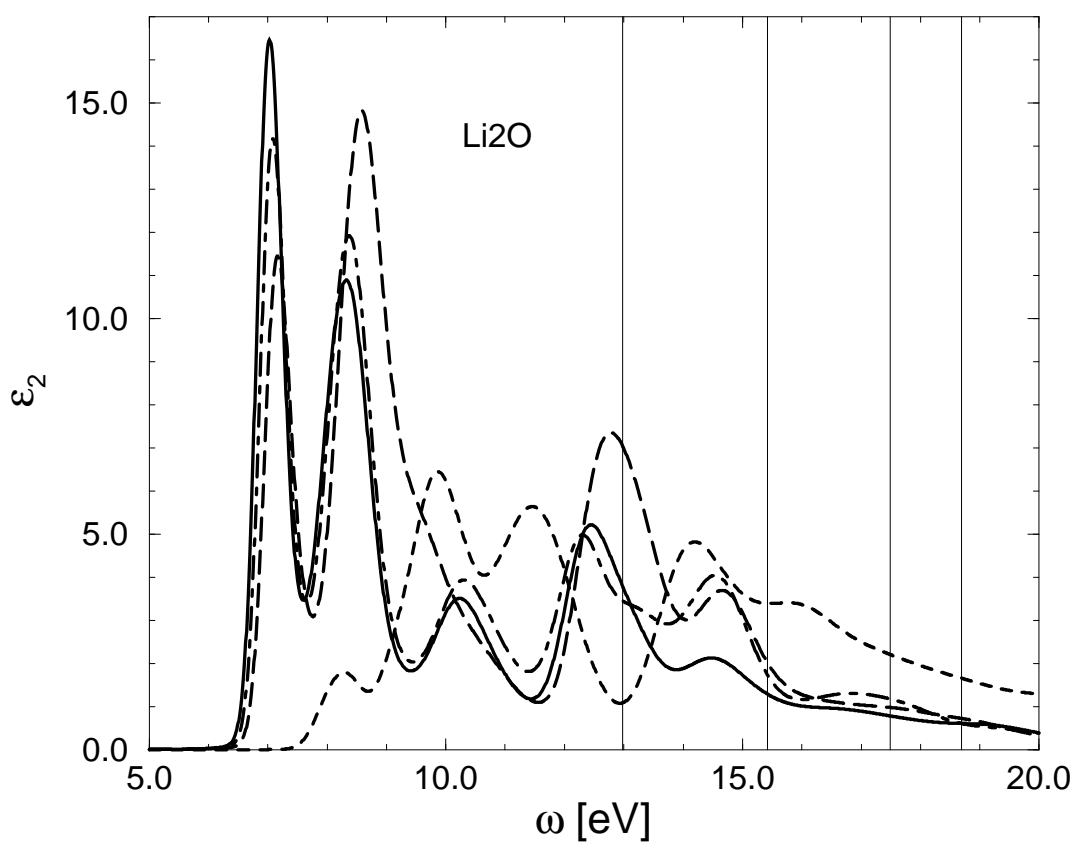

Figure 6: Absorption spectrum of lithium oxide. Short-dashed curve: calculation, no electron-hole interaction included. Continuous curve: full exciton calculation. Long-dashed curve: first order result, block size 300. Dot-dashes curve: first order result, block size 700 . Vertical bars: block cuts

\section{$\underline{\mathrm{Li}_{2} \mathrm{O}}$}

Another insulator of interest for our purpose is lithium oxide, $\mathrm{Li}_{2} \mathrm{O}$. $\mathrm{Li}_{2} \mathrm{O}$ is a material which is experimentally studied essentially because of its potential importance in the atomic energy domain. Several spectroscopic measurements are published in the literature [27], often focusing on the characterisation of radiation defects. Recently, there have been theoretical [7] and experimental [28] studies devoted to the question of excitonic effects at the band edge of $\mathrm{Li}_{2} \mathrm{O}$. In Ref. [7] a lowering of the optical gap due to excitonic effects of the order of $1 \mathrm{eV}$ was predicted. The experiment of Ref. [28] seems to be in agreement with this finding. It is hence interesting, on one side, to study excitonic effects in $\mathrm{Li}_{2} \mathrm{O}$ not only at the absorption onset, but over a $20 \mathrm{eV}$ range, and to compare with experiment. On the other hand, the fact that now also the transition energies are heavily affected by the electron-hole interaction presents an additional test for our approach. We have performed the ground state calculations using pseudopotentials of the Trouiller-Martins type [22], a plane-wave cutoff of $80 \mathrm{Ry}$, and using 2 special points in the irreducible part of the BZ. The theoretical lattice constant is $4.534 \mathrm{~A}$ in good agreement with the experimental value of $4.573 \mathrm{~A}$. We have then determined the GW corrections to the DFT-LDA eigenvalues, using up to 541 reciprocal lattice vectors and 280 bands. The correction to the direct LDA gap of $5.6 \mathrm{eV}$ turns out to be $2.3 \mathrm{eV}$, again with corrections which are slightly larger at the zone boundaries, in good agreement with Ref. [7].

Our independent quasiparticle absorption spectrum, calculated using 256 shifted k-points in the BZ and the first 9 bands, (short-dashed curve in Fig. 6) consequently shows an onset at about $7.9 \mathrm{eV}$, which well compares with the value of $7.99 \mathrm{eV}$ estimated in Ref. [28] for the band-gap energy, and is considerably higher than the measured onset at about $6.5-7$ $\mathrm{eV}$ [29, 28, 27]. We have then calculated the full exciton absorption spectrum according to equation (6), using the same parameters as for the independent quasiparticle spectrum. The 


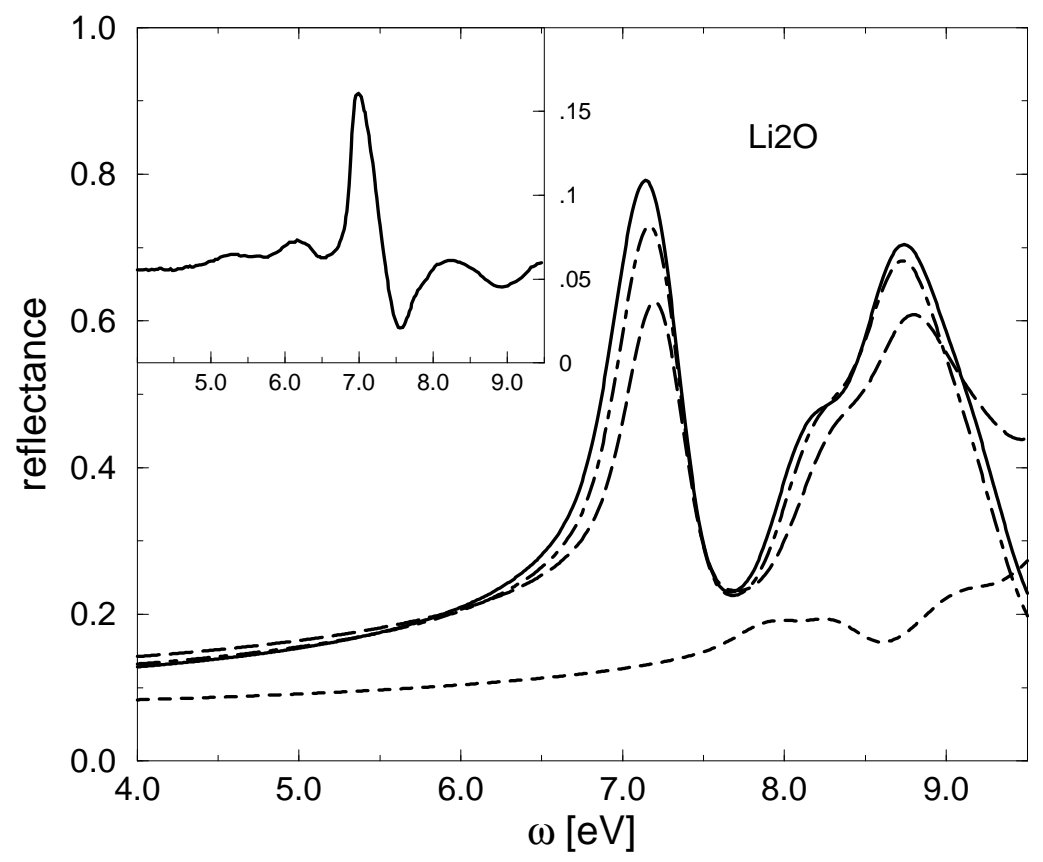

Figure 7: Reflectance spectrum of lithium oxide. Inset: experiment [28]. Short-dashed curve: calculation, no electron-hole interaction included. Continuous curve: full exciton calculation. Long-dashed curve: first order result, block size 300. Dot-dashed curve: first order result, block size 700 .

result is given by the continuous curve in Fig. 6. The onset has now been lowered, to about 7 $\mathrm{eV}$, which agrees well with the experiment and with the prediction of Ref. [7]. Moreover, as in the case of silicon and $\mathrm{MgO}$, considerable oscillator strength has been transferred to lower energies, giving rise to a sharp peak at the onset. This behaviour is similar to what has been found for $\mathrm{LiF}$ in Ref. [10]. Our calculated reflectance spectrum, given by the continuous curve in Fig. 7, reproduces the main features of the experimental result [28] (see inset Fig. (7), with a first strong peak at $7 \mathrm{eV}$, a second broader peak above $8 \mathrm{eV}$ and a sharp minimum between the two structures. A detailed analysis will be necessary in order to check whether the small structure on the low energy shoulder of the $8 \mathrm{eV}$-peak is related to the shoulder found experimentally, and whether the excitonic origin of this structure, proposed in Ref. [28], can be confirmed.

For this work, we concentrate on the question whether our perturbative approach can also be applied to this system with large excitonic binding energy. We have therefore also calculated the first order spectrum, given by the long-dashed curve in Fig. 6. Here the block size has been chosen to be $300 \times 300$. The essential part of the excitonic effect is already included in this spectrum. In particular, the binding energy of the first peak is given correctly. As mentioned above, we can improve this result systematically by increasing the block size: The dot-dashed curve has been calculated with a block size of $700 \times 700$. The vertical bars are the corresponding block cuts. The result is now in very good agreement with the full one. The same holds for the reflectance spectrum, as can be seen from the short-dashed $(\mathrm{GW})$, long-dashed $(300 \times 300)$ and dot-dashed $(700 \times 700)$ curves in Fig.7. This means that, even in a very unfavourable situation, our approach is advantageous.

\section{OUTLOOK}

We should stress the fact that up to now we have concentrated our effort only at the aim to save memory. It is however obvious that we can in principle use the same method also to 
save considerable CPU time and disk storage requirements: the biggest part of the exciton Hamiltonian $H^{e x c}$ is now not longer used in a direct diagonalization, but, for the calculation of $A_{\lambda}$, in the perturbation formula

$\sum_{t} H_{t \lambda}^{e x c} /\left(E_{\lambda}-E_{t}\right) A_{t}^{(0)}$, with $H_{t t^{\prime}}^{e x c}=\sum_{(v c),\left(v^{\prime} c^{\prime}\right)} A_{t}^{*(0)(v c)} H_{(v c)}^{e x c,\left(v^{\prime} c^{\prime}\right)} A_{t^{\prime}}^{(0)\left(v^{\prime} c^{\prime}\right)}$. The fact that now $H$ acts on a vector, instead of being inverted or diagonalized, allows to change space for each part of the Hamiltonian separately. In particular, one can go back to real space for the electron-hole attraction part $W$, which, according to Eq. (3), is then diagonal in two of the four coordinates. In this way, our approach will hence combine both the advantages of the effective two-particle picture used in [6, 8, 11], and of the recursive inversion approach proposed in [9, 10].

\section{CONCLUSIONS}

In conclusion, we have analysed in detail the nature of the excitonic effects in different materials. This has allowed us to design a method which improves the efficiency of the $a b$ initio calculation of optical spectra including the electron-hole interaction. By using a perturbative approach, the method avoids the diagonalisation of large matrices and therefore has the advantage of significant memory saving. Still, it is ab initio in the sense that one can converge the results smoothly to the exact ones, by a controllable parameter (i.e. the block size).

The method works extremely well for systems with weak interaction, but strong excitonic effects, as we have shown for the case of bulk silicon. We can therefore expect that it will be of great usefulness for the study of systems like those, for example, built up of many silicon atoms (e.g. amorphous silicon), where we are in the ideal condition of weak interaction but large density of transitions.

We have tested the approach also for unfavourable cases, using as examples two insulators. In spite of the strongly increased interaction strength, we can still obtain good spectra in the gap region, with memory savings of two orders of magnitude. We have also shown that the method yields valid results for the real part, not only the imaginary part, of $\epsilon_{M}$, therefore allowing for the description of spectra like the reflectance. The inclusion of first order corrections to a given subset of states is hence a very promising method in order to obtain, with little effort, improved spectra in the low energy region.

\section{ACKNOWLEDGEMENTS}

For this work, computer time was granted by IDRIS (Project No. CP9/990544).

\section{References}

[1] P. Hohenberg and W. Kohn, Phys. Rev. 136, B864 (1964); W. Kohn and L. J. Sham, Phys. Rev. 140, A1133 (1965).

[2] L. Hedin, Phys. Rev. A 139, 796 (1965).

[3] M. S. Hybertsen and S. G. Louie, Phys. Rev. Lett. 55, 1418 (1985); Phys. Rev. B 34, 5390 (1986).

[4] R. W. Godby, M. Schlüter, and L. J. Sham, Phys. Rev. Lett. 56, 2415 (1986); Phys. Rev. B 37, 10159 (1988).

[5] W. Hanke and L. J. Sham, Phys. Rev. Lett. 43, 387 (1979); Phys. Rev. B 21, 4656 (1980).

[6] G. Onida, L. Reining, R. W. Godby, R. Del Sole, and W. Andreoni, Phys. Rev. Lett. 75, 818 (1995). 
[7] S. Albrecht, G. Onida, and L. Reining, Phys. Rev. B 55, 10278 (1997).

[8] S. Albrecht, L. Reining, R. Del Sole, and G. Onida, Phys. Rev. Lett. 80, 4510 (1998).

[9] L. X. Benedict, E. L. Shirley, and R. B. Bohn, Phys. Rev. B 57, R9385 (1998).

[10] L. X. Benedict, E. L. Shirley, and R. B. Bohn, Phys. Rev. Lett. 80 , 4515 (1998).

[11] M. Rohlfing and S. G. Louie, Phys. Rev. Lett. 80 , 3320 (1998).

[12] L. X. Benedict and E. L. Shirley, Phys. Rev. B 59, 5441 (1999).

[13] M. Rohlfing and S. G. Louie, Phys. Rev. Lett. 81, 2312 (1998); Phys. Rev. Lett. 82, 1959 (1999).

[14] W. Hanke and L. J. Sham, Phys. Rev. B 12, 4501 (1975); R. Del Sole and E. Fiorino, Phys. Rev. B 29, 4631 (1984).

[15] G. Strinati, Phys. Rev. Lett. 49, 1519 (1982); Phys. Rev. B 29, 5718 (1984).

[16] F. Bechstedt, K. Tenelsen, B. Adolph, and R. Del Sole, Phys. Rev. Lett. 78, 1528 (1997).

[17] H. J. Monkhorst and J. D. Pack, Phys. Rev. B 13, 5188 (1976).

[18] S. Albrecht, L. Reining, G. Onida, V. Olevano, and R. Del Sole, Phys. Rev. Lett. 83, 3971 (1999).

[19] P. Lautenschlager, M. Garriga, L. Viña, and M. Cardona, Phys. Rev. B 36, 4821 (1987).

[20] M. Rohlfing and S. G. Louie, in Electron Correlations and Materials Properties, edited by. A. Gonis, N. Kioussis, and M. Ciftan (Kluwer Academic/Plenum, New York, 1999), p. 309.

[21] R. Haydock, Comput. Phys. Commun. 20, 11 (1980).

[22] N. Troullier and J. L. Martins, Phys. Rev. B 43, 1993 (1991).

[23] U. Schoenberger and F. Aryasetiawan, Phys. Rev. B 52, 8788 (1995).

[24] E. L. Shirley, Phys. Rev. B 58, 9579 (1998).

[25] D. M. Roessler and W. C. Walker, Phys. Rev. 159, 733 (1967).

[26] In order to discuss the remaining discrepancies, we would have to converge the spectrum better, especially what the BZ sampling is concerned. This is however beyond the scope of this work.

[27] K. Noda, Y. Ishii, H. Ohno, H. Watanabe, and H. Matsui, Adv. Ceram. Mater. 25, 155 (1989); N. M. Masaki, K. Noda, H. Watanabe, R. G. Clemmer, and G. W. Hollenberg, J. Nucl. Mater. 212-215, 908 (1994); F. Beuneu and P. Vajda, Phys. Rev. Lett. 76, 4544 (1996); F. Beuneu, P. Vajda, and O. J. Zogal, Colloids and Surfaces A 158, 83 (1999); K. Uchida, K. Noda, T. Tanifuji, S. Nasu, T. Kirihara, and A. Kikuchi, Phys. Stat. Sol. (a) 58, 557 (1980); L. Liu, V. E. Henrich, W. P. Ellis, and I. Shindo, Phys. Rev. B 54, 2236 (1996); T. Osaka and I. Shindo, Solid State Commun. 51, 421 (1984).

[28] Y. Ishii, J. Murakami, and M. Itoh, J. of the Physical Society of Japan 68, 696 (1999).

[29] W. Rauch, Z. Phys. 116, 652 (1940). 